\title{
From plastic to elastic stress relaxation in highly mismatched $\mathrm{SiGe/Si}$ heterostructures
}

Fabio Isa $^{\mathrm{a}, \uparrow, *}$, Marco Salvalaglio ${ }^{\mathrm{b}}$, Yadira Arroyo Rojas Dasilva ${ }^{\mathrm{c}}$, Arik Jung ${ }^{\mathrm{a}, \dagger}$, Giovanni Isella ${ }^{\mathrm{d}}$, Rolf Erni $^{\mathrm{c}}$, Philippe Niedermann ${ }^{\mathrm{e}}$, Pierangelo Gröning ${ }^{\mathrm{c}}$, Francesco Montalenti ${ }^{\mathrm{b}}$ and Hans von Känel ${ }^{\mathrm{a}, \dagger}$

${ }^{a}$ Laboratory for Solid State Physics, ETH Zürich, Otto-Stern-Weg 1, 8093, Zürich, Switzerland

${ }^{\mathrm{b}}$ L-NESS and Department of Materials Science, Università di Milano-Bicocca, Via Cozzi 55, 20125, Milano, Italy

${ }^{c}$ Electron Microscopy Center Empa, Swiss Federal Laboratories for Materials Science and Technology, Überlandstrasse 129, 8600, Dübendorf, Switzerland

${ }^{\mathrm{d}}$ L-NESS and Department of Physics, Politecnico di Milano, Via Anzani 42, 22100, Como, Italy

${ }^{\mathrm{e}}$ CSEM, Rue Jaquet-Droz 1, 2002, Neuchâtel, Switzerland

* Corresponding author email address: isaf@phys.ethz.ch

$\uparrow$ Present address: Electron Microscopy Center Empa, Swiss Federal Laboratories for Materials Science and Technology, Überlandstrasse 129, 8600, Dübendorf, Switzerland

\section{Abstract}

We present a detailed experimental and theoretical analysis of the epitaxial stress relaxation process in micro-structured compositionally graded alloys. We focus on the pivotal $\mathrm{SiGe} / \mathrm{Si}(001)$ system employing patterned Si substrates at the micrometre-size scale to address the distribution of threading and misfit dislocations within the heterostructures. SiGe alloys with linearly increasing Ge content were deposited by low energy plasma enhanced chemical vapour deposition resulting in isolated, tens of micrometre tall 3D crystals. We demonstrate that complete elastic relaxation is achieved by appropriate choice of the Ge compositional grading rate and Si pillar width. We investigate the nature and distribution of dislocations along the [001] growth direction in SiGe crystals by transmission electron microscopy, chemical defect etching and etch pit counting. We show that for $3 \mu \mathrm{m}$ wide $\mathrm{Si}$ pillars and a Ge grading rate of $1.5 \% \mu^{-1}$, only misfit dislocations are present while their fraction is reduced for higher Ge grading rates and larger structures due to dislocation interactions. The experimental results are interpreted with the help of theoretical calculations based on linear elasticity 
theory describing the competition between purely elastic and plastic stress relaxation with increasing crystal width and Ge compositional grading rate.

\section{Keywords}

Graded buffer; elastic relaxation; dislocations; epitaxy; SiGe

\section{Introduction}

The monolithic integration of dissimilar semiconductors on Si has a fundamental role in the development of devices which benefit from the reliable Si CMOS technology in combination with other material properties [1], [2], [3]. Unfortunately, if the deposited semiconductor has a lattice parameter differing from the one of the substrate, misfit and threading dislocations (MDs, TDs) are introduced to release the epitaxial stress after a certain critical thickness [4], [5]. These extended defects have detrimental impacts on the properties of integrated devices [6], and therefore their control and reduction is a priority in the field of heteroepitaxy.

In the last decades several approaches have been employed to reduce the density of TDs in mismatched layers. A few examples are annealing treatments to improve the mobility and interaction probability of TDs [7], [8], aspect ratio trapping, where the epitaxial material is selectively deposited in oxide mask windows and TDs are geometrically confined close to the heterointerface [9], [10], [11], pendeoepitaxy [12] and 3D heteroepitaxy [13], [14], [15].

One of the most employed and successful methods to reduce the TD density in SiGe and III-V layers is the epitaxial growth of compositional graded alloys [16], [17]. This method is based on the epitaxial growth of alloys whose composition is continuously varied during the deposition (for $\mathrm{Si}_{1-x} \mathrm{Ge}_{x} / \mathrm{Si}$

materials the $x$ increment is typically $\left.\sim 10 \% \mu^{-1}[18]\right)$. This process assures a small residual epitaxial strain which favours the gliding of existing dislocations through the entire thickness of the epitaxial layer. This method reduces the probability of dislocation interaction and pinning which usually leads to 
the nucleation of new dislocation loops and higher TD density.

Another solution to reduce the density both of TDs and MDs is to use compliant nanopatterned substrates which permit the epitaxial stress to be released elastically, thus avoiding plastic relaxation. According to the work of Luryi and Suhir [19], Atkinson et al. [20], Zubia et al. [21] coherent mismatched crystals can be deposited on substrates patterned in the form of mesa structures with widths below $\sim 15$ times the critical thickness for plastic relaxation. The epitaxial stress is partitioned between the epitaxial material and the patterned substrate and fully relaxed elastically. Indeed dislocation-free Ge/Si nanocrystals have been achieved on Si nanotips [22], [23]. Zhou et al. [24] predicted on the other hand that coherent $\mathrm{CdTe} / \mathrm{CdS}$ heterostructures up to $90 \mathrm{~nm}$ in width can be obtained by compositional grading.

Recently we demonstrated [25] completely dislocation-free highly mismatched heterostructures with lateral widths at the micrometre scale. This was achieved by combining the compositional grading approach with the epitaxial growth of isolated 3D SiGe crystals with high aspect-ratios [13] on micrometre-sized Si pillars by low energy plasma enhanced chemical vapour deposition (LEPECVD) [26]. The high aspect ratio of the SiGe heterostructures and a shallow Ge grading rate (GR) of $1.5 \%$ $\mu \mathrm{m}^{-1}$ lead to full elastic relaxation of the epitaxial stress, as indicated also by theoretical calculations based on a model published in Ref. [27].

In this work we address the plastic relaxation mechanism in compositional graded alloys and study the density and nature of dislocations as a function of the Ge GR and the Si pillar width, which are both key factors for the elastic relaxation mechanism. In particular, we performed defect etching experiments to reveal the distribution of dislocations along the [001] direction of the SiGe crystals. We discriminated between TDs and MDs by looking at the shapes of the etch pits with scanning electron microscopy (SEM). The extensive and statistically relevant results obtained by defect etching and SEM are supported by transmission electron microscopy (TEM) measurements and theoretical calculations 
of the MD formation energy.

\section{Materials and Methods}

\subsection{Epitaxial growth of SiGe alloys by LEPECVD}

SiGe alloys were deposited by LEPECVD [26] on deeply patterned 4" Si(001) substrates (n-type, 3 - 7

$\Omega \mathrm{cm})$. The patterning consisted of pillars of various sizes arranged in $8 \times 8 \mathrm{~mm}^{2}$ areas, separated by a 2 mm wide planar rim. The Si pillars have square shapes with sides aligned along the $<110>$ crystal directions and are $8 \mu \mathrm{m}$ tall. Their width ranges from 2 to $50 \mu \mathrm{m}$ and the width of the separating gap is $4 \mu \mathrm{m}$. Additional information about the preparation and cleaning prior to the epitaxial growth on patterned Si substrates can be found in Ref. [28].

The structure of compositionally graded crystals consists of: i) an $8 \mu$ m thick $\operatorname{Si}_{1-x} \mathrm{Ge}_{x}$ alloy with constant Ge content $x=0.5 \%$, ii) a compositionally graded part with linearly increasing Ge content at a rate of either $1.5,3$ or $6 \% \mu \mathrm{m}^{-1}$ and iii) a $1 \mu \mathrm{m}$ thick $\mathrm{Si}_{0.6} \mathrm{Ge}_{0.4}$ capping layer. The initial growth temperature of $750{ }^{\circ} \mathrm{C}$ for $0<x<0.1$ was linearly decreased with increasing $x$ to the final value of 590 ${ }^{\circ} \mathrm{C}$. The $\mathrm{Si}_{1-x} \mathrm{Ge}_{x}$ alloy growth rate changed from $5.9 \mathrm{~nm} / \mathrm{s}$ to $6.2 \mathrm{~nm} / \mathrm{s}$ between $x=0$ and $x=0.4$. These growth conditions ensure a surface roughness below $3 \mathrm{~nm}$ (measured in the planar regions) and vertical growth of isolated SiGe crystals [28]. The Ge content in the compositionally graded part was increased in steps of $0.5 \%$. A sample consisting only of an $8 \mu \mathrm{m}$ thick $\mathrm{Si}_{1-x} \mathrm{Ge}_{x}$ alloy with constant Ge content $x=$ $0.5 \%$ is also considered for the dislocation analysis. 


\subsection{Defect etching of SiGe crystals}

The dislocation distribution in SiGe crystals was addressed by cleaving each pattern along the [110] direction, dipping into a $\mathrm{Cr}$ based defect etching solution and counting the etch pits appearing on the $\{110\}$ sidewalls of the crystals by SEM.

The $\mathrm{Cr}$ based defect etching solution consisted of $\mathrm{CrO}_{3}(10.8 \mathrm{~g})+\mathrm{HF} 40 \%(110 \mathrm{ml})+$ deionized water (113 ml) kept at a temperature of $0{ }^{\circ} \mathrm{C}$. The etching time varied between $240 \mathrm{~s}$ to $420 \mathrm{~s}$.

Etch pit counting on crystals occupying an equivalent area from $7 \times 10^{3}$ to $2 \times 10^{4} \mu^{2}$ ensured reliable statistics. This procedure of counting dislocation related etch pits is substantially different from the standard technique where only TDs emerging at the top surface of heterostructures can be detected. With our approach we evaluated the absence of dislocations in SiGe crystals (complete elastic relaxation of the epitaxial stress), discriminated between MDs and TDs, assessed their distribution within the graded structure, and observed the injection of dislocations into the Si substrate. The error on the dislocation density in SiGe crystals is considered $\pm 20 \%$ due to the uncertainty in counting overlapping etch pits, distinguishing an etch pit from a surface contamination and irregular crystal surfaces. In our work, the dislocation density of planar layers and of crystals, always refers to dislocations revealed by cross-sectional SEM images on the $\{110\}$ planes. The dislocation density of SiGe crystals was evaluated by considering only the equivalent surface of $\{110\}$ sidewalls while neglecting the gap between neighbouring crystals.

\subsection{Transmission electron microscopy analysis}

TEM measurements were performed by means of a Philips CM30 microscope operated at $300 \mathrm{kV}$ to evaluate the ratio between MDs and TDs in SiGe crystals grown with a Ge GR $=1.5 \% \mu^{-1}$ on $5 \times 5$ 
$\mu \mathrm{m}^{2} \mathrm{Si}$ pillars. The samples were prepared by mechanical polishing, dimple grinding and ion milling with Ar at $3 \mathrm{kV}$.

The MD ratio is defined as the number of MDs divided by the total dislocations number in a certain area. Both MDs with dislocation line along the [110] and [110] directions were taken into account. By considering the electron beam propagating along the [110] direction, MDs with dislocation line along the [110] and [1ํㅣ directions would appear as spots and segments, respectively. If the sample is rotated around the [001] and [11̄0] directions to obtain the two-beam conditions, MDs along [110] would look like short segments with the orientation changing by $90^{\circ}$ from [110] to [001], respectively. In contrast, the dislocation line projection of MDs along [1ํㅣ] is barely affected. Dislocations with lines inclined to the (001) plane were considered to be TDs.

Three different SiGe crystals on $5 \times 5 \mu \mathrm{m}^{2}$ Si pillars were investigated in the area close to the $\mathrm{Si}$ heterointerface. Compared to the defect etching analysis where the shape of an etch pit could be influenced by other surrounding defects causing overlapping of pits (see figure 5 and related discussion), the TEM analysis is more precise in determining the misfit or threading nature of dislocations. On the other hand due to the limited statistics of TEM compared to defect etching, different values of the MD ratio are expected.

\subsection{Dislocation energy calculations}

In order to support the interpretation of the experimental results, elasticity-theory calculations were performed aimed at understanding when the total elastic energy of a $\mathrm{SiGe/Si}$ heterostructure is lowered by the presence of a MD, thus promoting its nucleation. To this end, we adopted a 2D model where the linear elasticity theory equations were numerically solved by the finite element method (FEM). Even though this $2 \mathrm{D}$ approach is simplified it provided elastic fields compatible with the complete crystal 
relaxation of high aspect-ratio vertical structures when 3D calculations are considered [27]. As

illustrated in figure 1, the 2D system consisted of a slice of the $\mathrm{SiGe/Si}$ crystal in the $x z$-plane with axis along $\widehat{\boldsymbol{X}}=[110], \widehat{\boldsymbol{Y}}=[\overline{1} 10]$ and $\widehat{\boldsymbol{Z}}=[001]$. The vertical SiGe/Si structure was modelled as a simple parallelepiped, with height-to-base aspect ratio obtained from SEM images.

At variance with previous investigations (see Ref. [25]), an $8 \mu \mathrm{m}$ thick alloy made of almost pure Si (Ge content $x=0.5 \%$ ) was firstly grown to reduce the gap between neighbouring crystals preventing further increase of the SiGe crystals width during their compositionally graded part and to limit $\mathrm{SiGe}$ deposition on the Si pillar sidewalls. Indeed as indicated in figure $2 b$ ) the width of the bottom and the top graded SiGe structure, labeled $L_{1}$ and $L_{2}$, respectively, differs only slightly with $L_{1}=8.25 \mu \mathrm{m}$ and $L_{2}=9.05 \mu \mathrm{m}$. The calculations (see details below) were performed by considering a constant width $L=$ $L_{2}$. Since the elastic budget for strain relief is expected to increase with increasing crystal width [27], we overestimated the tendency to insert a MD in the actual structure since its real width varies between $L_{1}$ and $L_{2}$. The Si substrate was chosen five times taller and wider than the Si pillar (see figure 1) [27]. Within this $2 \mathrm{D}$ system we calculated the $\mathrm{MD}$ formation energy as $\Delta E=E_{M D}-E_{c o h}$, where $E_{M D}$ and $E_{c o h}$ are the elastic energies computed from the elastic fields at equilibrium [29] with and without a MD, respectively. The elastic fields were calculated as reported in Refs. [30],[25]. Accordingly, the mechanical equilibrium condition $\nabla \cdot \boldsymbol{\sigma}[\mathbf{u}]=0$, where $\boldsymbol{\sigma}[\mathbf{u}]$ is the stress field and $\mathbf{u}$ the displacement field, was solved considering the proper boundary conditions (BCs). $E_{c o h}$ was calculated assuming as initial value of $\boldsymbol{\sigma}$ the heteroepitaxial (eigen)stress $\sigma_{i j}=\left[\sigma_{\mathrm{het}}\right]_{i j}=x \delta_{i j} C_{i j}\left(a_{\mathrm{Si}}-a_{\mathrm{Ge}}\right) / a_{\mathrm{Ge}}$ where $C_{i j}$ is the stiffness tensor, $x$ the Ge content, and $a_{\mathrm{Ge}}$ and $a_{\mathrm{Si}}$ the lattice parameters of $\mathrm{Ge}$ and $\mathrm{Si}$, respectively. As sketched in figure 1 the compositionally graded part was modelled by considering the crystal to be composed of $N=80 \mathrm{Si}_{1-x} \mathrm{Ge}_{x}$ layers with increasing Ge content $x$ from $0.5 \%$ to $40 \%$ in steps of $\Delta x=0.5 \%$. The thickness of each $N$-layer $\left(t_{N}\right)$ is calculated from the Ge GR: $t_{N}=\Delta x / \mathrm{GR}$. Isotropic elastic constants were interpolated according to Vegard's law as reported in Ref. [25]. $E_{M D}$ 
was computed by imposing as initial (eigen)stress condition $\boldsymbol{\sigma}=\boldsymbol{\sigma}_{\text {het }}+\boldsymbol{\sigma}_{\text {dislo }}$ where $\boldsymbol{\sigma}_{\text {dislo }}$ is the stress tensor generated by a $60^{\circ} \mathrm{MD}$ in the bulk [31] with its line parallel to $\widehat{\boldsymbol{Y}}$ and Burgers vector $b / \sqrt{2}$ [ $\left.\begin{array}{lll}1 & 0 & 1\end{array}\right]$ where $b=0.385 \mathrm{~nm}$. The singularity of the stress field at the dislocation core is removed by exploiting the regularization introduced in Ref. [32]. Note that this approach also allows the core energy to be effectively included by tuning the single additional parameter $a$, which quantifies the extension of the region affected by the regularization. Here we take the value $a=0.1218 \mathrm{~nm}$ already used in previous works [33]. The adopted BCs are illustrated in figure 1 . The free surface BC $\boldsymbol{\sigma}[\mathbf{u}] \cdot \widehat{\mathbf{n}}$ $=0$ was considered at the surfaces of the vertical $\mathrm{SiGe} / \mathrm{Si}$ structure and at the top surface of the $\mathrm{Si}$ substrate, with $\widehat{\mathbf{n}}$ denoting the normal of the considered free surface. The fixed $\mathrm{BC} \mathbf{u}=0$ was considered at the bottom of the Si substrate. The gliding BC $u_{x}=0$ was set at the lateral sidewalls of the Si substrate to mimic the periodicity along the [110] direction (however, we verified that lateral interactions are negligible due to the large simulation cell).

The tendency to insert a MD dislocation is evaluated by the sign of $\Delta E$. If $\Delta E>0$ the insertion of a MD increases the elastic energy, so that the system is predicted to be lattice coherent. Conversely, if $\Delta E<0$, plasticity sets in as the MD insertion decreases the elastic energy of the system. Notice that this criterion is based on the elastic energy only, so that it evaluates the competition between elasticity and plasticity on a thermodynamic basis. Some delay with respect to the thermodynamically predicted onset of plasticity should always be expected due to nucleation barriers.

In order to evaluate the actual MD formation energy, one should compute the minimum of $\Delta E$ called $\Delta E_{\text {min }}$ for a given crystal configuration. The parameter space to be sampled in the minimization involves the position of the dislocation along all the interfaces between different layers labelled by the index $i$, accounting also for the increasing number $N$ of the layers with different Ge content during the growth (see also figure 1). In this work $\Delta E_{\min }$ was computed by varying the dislocation position along the $\widehat{X}=[110]$ axis at the $i=0$ interface to assess the coherency of the SiGe $x=0.5 \%$ alloy and at the $i$ 
$=1$ interface to investigate the relaxation of the compositionally graded crystals. The latter was investigated for different $N$ values. Interfaces with $i>1$ were not considered as we demonstrated in Ref. [25] that the minimum of the formation energy is always found very close to the first interface. Even if the energy minimum is obtained at the interface with $i>1$, the difference with respect to the calculation at $i=1$ is negligible.

\section{Results and discussions}

\subsection{Misfit and threading dislocation distribution in SiGe crystals}

The epitaxial growth of compositional graded SiGe alloys on patterned $\mathrm{Si}(001)$ substrates by LEPECVD resulted in isolated and faceted SiGe crystals as indicated in figure 2a) and b). The top crystal facets, which are visible in the SEM image of figure 2a), consist of a central (001) surface and 4-fould symmetric $\{113\}$ and $\{111\}$ facets close to the edges. The vertical sidewalls shown in the cross-sectional SEM image of figure $2 b)$ are $\{110\}$ surfaces. The growth mechanism and the facet distribution of such SiGe crystals have been already described in Ref. [13], [28].

Figure 2c) shows a cross-sectional SEM image of a SiGe crystal $\left(\mathrm{GR}=1.5 \% \mu \mathrm{m}^{-1}\right)$ after defect etching. Etch pits related to emerging dislocations are visible both in the SiGe alloy and in the Si substrate pillar.

By averaging over dozens of crystals (section 2.2), the probability distribution of the dislocation density was obtained for the $\mathrm{Si}_{1-x} \mathrm{Ge}_{x}$ alloy with a constant composition of $x=0.5 \%$ and for $\mathrm{SiGe}$ crystals grown with different Ge GR on Si pillars 2, 3, 5, 10 and $50 \mu \mathrm{m}$ in width. The results are shown in figure 3.

Figure 3a) indicates that independently of the Si pillar width (up to a Si pillar width of $50 \mu \mathrm{m}$ ), more than $95 \%$ of the analysed SiGe crystals consisting only of the $8 \mu \mathrm{m}$ thick constant composition alloy 
with $x=0.5 \%$, are completely free from dislocations. This finding indicates that the SiGe crystals release the epitaxial stress elastically. In the unpatterned area, where the elastic relaxation mechanism is negligible, dislocations were found with a density of $(4.4 \pm 2.3) \times 10^{6} \mathrm{~cm}^{-2}$. Calculations of the MD nucleation energy for the constant composition $\mathrm{SiGe} x=0.5 \%$ crystals perfectly agree with the experimental data. Indeed, positive $\Delta E_{\min }$ values were obtained when the dislocation is placed at the interface of the $\mathrm{SiGe} / \mathrm{Si}$ system $(i=0)$ for all Si pillar widths (here sampled up to $L=10 \mu \mathrm{m})$. In contrast, as shown in figure $3 \mathrm{~b}$ ), the dislocation distribution in $\mathrm{Si}_{1-x} \mathrm{Ge}_{x}$ crystals, consisting of the $x=$ $0.5 \%$ alloy plus the compositionally graded part up to $x=40 \%$ with a Ge GR $=1.5 \% \mu^{-1}$, strongly depends on the Si pillar width. In the following discussion the dislocation density includes both the constant composition $(x=0.5 \%)$ and the graded part. All the SiGe crystals on $2 \times 2 \mu \mathrm{m}^{2}$ Si pillars have zero dislocations appearing on the $\{110\}$ sidewalls (within the investigated ensemble, see section 2.2), indicating purely elastic relaxation of the epitaxial stress [25]. By increasing the Si pillar width to 3 and $5 \mu \mathrm{m}, \mathrm{SiGe}$ crystals have only a $30.2 \%$ and a $5.9 \%$ probability, respectively, to be dislocation-free. Therefore the elastic relaxation mechanism strongly depends on the Si pillar width which determines the SiGe crystals' aspect ratio. By further increasing the Si pillar width to 10 and $50 \mu \mathrm{m}$, all SiGe crystals contain dislocations and the average of the dislocation density distribution is shifted towards increasingly higher values. This finding indicates that the elastic relaxation mechanism gets less and less effective for larger width of the Si pillars.

Calculations of the MD formation energy perfectly match the experimental results. Indeed positive values of $\Delta E_{\min }$, and therefore complete lattice coherence, were obtained for SiGe crystals deposited on $2 \times 2 \mu \mathrm{m}^{2}$ Si pillars $(L=5.9 \mu \mathrm{m})$ at a Ge GR $=1.5 \% \mu \mathrm{m}^{-1}$, as indicated by the solid black line in figure 4. Conversely, negative values of $\Delta E_{\min }$ were calculated by increasing the size of the SiGe crystals on $3 \times 3 \mu \mathrm{m}^{2}$ Si pillars $(L=7 \mu \mathrm{m})$ for the same Ge GR $=1.5 \% \mu \mathrm{m}^{-1}$ as indicated by the dashed black line in figure 4. According to the negative sign of the MD formation energy, that system is predicted to 
contain dislocations. SiGe crystals deposited on larger Si pillars showed even lower MD formation energy, indicating a stronger tendency towards plasticity after the deposition of the first $\mathrm{Si}_{1-x} \mathrm{Ge}_{x}$ graded layers.

The results reported in figure $3 \mathrm{c}$ ) and d) demonstrate that by increasing the Ge GR to 3 and $6 \% \mu \mathrm{m}^{-1}$ respectively, the elastic relaxation mechanism is no longer effective even for SiGe crystals on the smallest Si pillars $2 \mu \mathrm{m}$ in width. Calculations of the MD formation energy agree with the experimental results, giving always negative values of the MD formation energy. As an example $\Delta E_{\text {min }}(N)$ for SiGe crystals deposited at a GR $=3 \% \mu \mathrm{m}^{-1}$ on $2 \times 2 \mu \mathrm{m}^{2}$ Si pillars $(L=5.9 \mu \mathrm{m})$ is shown in figure 4 by the dotted black line. Here $\Delta E_{\min }(N)$ becomes negative for $N>7$.

The average of the dislocation distribution in SiGe crystals, both for Ge GR $=3$ and $6 \% \mu^{-1}$, increases with increasing Si pillar width, as already explained before for Ge GR $=1.5 \% \mu \mathrm{m}^{-1}$. The dislocation density in the unpatterned area increased from $(9.0 \pm 1.7) \times 10^{6} \mathrm{~cm}^{-2}$ for Ge GR $=1.5 \% \mu \mathrm{m}^{-1}$, to $(7.1 \pm$ 1.4) $\times 10^{7} \mathrm{~cm}^{-2}$ for Ge GR $=3 \% \mu \mathrm{m}^{-1}$ and finally to $(7.4 \pm 1.7) \times 10^{6} \mathrm{~cm}^{-2}$ for Ge GR $=6 \% \mu \mathrm{m}^{-1}$. These values indicate that by incrementing the Ge GR the interaction and pinning of dislocations becomes more probable requiring the nucleation of new dislocation loops [34] which result in higher dislocation densities [35]. Similar results were found for the SiGe crystals. They are reflected by the shift of the average dislocation density towards larger values with increasing Ge GR for each Si pillar width. This observed trend is mostly ascribed to two concomitant effects: the plastic relaxation mechanism which gets more effective and interactions and pinning of dislocations which become more probable. Interestingly, for all Ge GR there is a certain probability to find SiGe crystals on $50 \times 50 \mu \mathrm{m}^{2} \mathrm{Si}$ pillars with a dislocation density higher than the corresponding one in the unpatterned area although the elastic relaxation mechanism is negligible in both cases. This result can be attributed to a more significant reduction of the TD density with the increase of the crystal thickness in the unpatterned area. Indeed since TDs lay in slanted $\{111\}$ planes, by incrementing the crystal thickness, the 
geometrical interaction, blocking and annihilation probability of threading segments [36] with opposite Burgers vector is enhanced. This TDs reduction mechanism is more effective in a continuous layer rather than in limited size SiGe crystals [9].

In order to obtain more information on the nature of dislocations in the SiGe crystals, a detailed analysis of the etch pit shape related to the emerging dislocations was performed. Indeed MDs and TDs can be distinguished thanks to the different shape of the associated etch pits as shown in figure 5a). The shapes of the etch pits differ because the dislocation line of a MD or a TD has a different inclination with respect to the $\{110\}$ sidewall of the SiGe crystals where they are observed. MDs have the dislocation line along the $\langle 110\rangle$ directions in (001) planes, resulting in 4-fold symmetric etch pits, one of which is indicated in light blue in figure 5b). On the other hand, TDs have the dislocation line lying in slanted $\{111\}$ planes, and create asymmetric and elongated etch pits such as the one marked in red in figure 5c). Similar results on the etch pit shape related to dislocations are reported in Refs. [37], [38] and [39]. MDs and TDs have not been distinguished in SiGe crystals on Si pillars $50 \mu \mathrm{m}$ in width and in the unpatterned area due to the large overlap of etch pits.

The MD fraction, defined as the number of MDs over the number of TDs + MDs, in SiGe crystals grown with different Ge GR as a function of the Si pillar width is reported in figure 5d). For Ge GR = $1.5 \% \mu \mathrm{m}^{-1}$ and Si pillar width of $3 \mu \mathrm{m}$, all the detected etch pits (within the experimental statistics) are related to MDs. This result indicates that dislocation loops can evolve towards the ideal configuration for strain release given by a misfit segment parallel to the heterointerface and running across the whole crystal. This is possible only when dislocation loops are free to slip, in the absence of any blocking interactions with other TDs [40]. This scenario can occur only for SiGe crystals with low dislocation density.

Indeed by increasing the Si pillar width to 5 and $10 \mu \mathrm{m}$ whereupon the dislocation density increases (see figure 3b)), the MD fraction is reduced to 70\% due to the appearance of TDs. The MD fraction 
in SiGe crystals with Ge GR $=1.5 \% \mu \mathrm{m}^{-1}$ on $5 \times 5 \mu \mathrm{m}^{2}$ Si pillars was measured also by TEM, yielding a value of $\sim 56 \%$ as indicated by the purple star in figure $5 \mathrm{~d}$ ). In view of the limited TEM statistics we consider the MD fractions obtained by the two techniques to be compatible.

Conversely, SiGe crystals with Ge GR $=3 \% \mu \mathrm{m}^{-1}$ on $2 \times 2 \mu \mathrm{m}^{2}$ Si pillars have a MD fraction of $\sim 70 \%$. This finding indicates that even for small Si pillar width MDs do not slip across the entire width of the crystals. Indeed, in this case a larger dislocation density is present as the elastic budget increases, producing an appreciable interaction between dislocations. For larger Si pillar width the MD fraction trend is similar to that one observed for crystals with $\mathrm{Ge} \mathrm{GR}=1.5 \% \mu \mathrm{m}^{-1}$, i.e. by increasing the $\mathrm{Si}$ pillar width the fraction of detected MDs on the $\{110\}$ sidewalls decreases because of the effects described before.

The MD fraction as a function of the Si pillar width is completely different for SiGe crystals grown with a Ge GR $=6 \% \mu \mathrm{m}^{-1}$ (grey dots in figure $5 \mathrm{~d}$ )) where it is practically constant at $\sim 37 \%$ independent of the Si pillar width. The reduction of the MD fraction is compatible with that observed by increasing the Ge GR from $1.5 \% \mu \mathrm{m}^{-1}$ to $3 \% \mu \mathrm{m}^{-1}$. Moreover it indicates that for a GR $=6 \% \mu \mathrm{m}^{-1}$ the dislocation density in SiGe crystals is large enough to have similar dislocation interaction probability and nucleation events for all Si pillars wider than $2 \mu \mathrm{m}$.

\subsection{Distribution of dislocations along the [001] growth direction}

The spatial distribution of misfit and threading dislocations in a constant-composition mismatched layer deposited on a substrate substantially differs from that of a compositionally graded layer. The former contains an array of MD segments located at the layer/substrate interface and bounded by TDs [41], while in the latter MDs are distributed along the [001] growth direction [42] because of the continuous increment of the lattice parameter.

We evaluated the dislocation distribution along the [001] direction by dividing the $\mathrm{SiGe} / \mathrm{Si}$ structure 
into 6 different regions as sketched in figure 6a). The first region consists of the Si pillar, the second one of the $x=0.5 \%$ constant composition part $(0<Z<8 \mu \mathrm{m})$ and the remaining four are identified by a Ge content changing by $\delta x=0.1$ from region to region in the compositionally graded part. The thickness of the latter depends on the Ge GR. In figure 6b), c) and d) the value of the Ge content $x$ shown on the bottom abscissa refers to the highest Ge content in each region. Analogously, the $Z$ values on the upper abscissa indicate the height at which the $\mathrm{Si}_{1-x} \mathrm{Ge}_{x}$ alloys with $x=0.5 \%, 0.1,0.2,0.3$ and 0.4 are located.

Figure 6a) shows that dislocations were found both in the Si pillars and in the $x=0.5 \%$ constant composition region (several other images of analogous $\mathrm{SiGe/Si}$ crystals indicate the same). These dislocations were introduced only after the growth of the compositionally graded structure as a consequence of its plastic relaxation. Indeed as reported in figure $3 \mathrm{a}$ ) if only the $x=0.5 \%$ constant composition alloy is deposited, more than $95 \%$ of the crystals are dislocation-free. Ordinarily in lowmisfit heterostructures dislocation nucleation is likely to proceed by multiplication via Frank-Read sources. Under these circumstances, the repulsive interaction between identical dislocations generated by a single source can push them deep into the substrate, as observed also in the Ref. [40], [43], [44]. The dislocation distribution along the [001] growth direction for SiGe crystals compositionally graded with a Ge GR of 1.5, 3 and $6 \% \mu^{-1}$ on Si pillars with different widths is reported in figure $6 \mathrm{~b}$ ), c) and d), respectively. We notice that by reducing the Si pillar width and/or by decreasing the Ge GR, dislocations are predominantly located in the regions of lowest Ge content. This is a direct signature of the role played by elastic relaxation in the regime where it is not effective enough to completely avoid dislocation nucleation. As the crystal width approaches the critical value allowing for complete lattice coherence, dislocations tend to be located close to the interface between the $x=0.5 \%$ alloy and the compositionally graded region. Indeed as already discussed in section 2.4 this region is the most energetically favoured one to introduce the first dislocation. Reducing the Ge GR has a similar effect. 
Indeed for a fixed Ge content $x$, the lower the Ge GR the taller is the crystal and the more efficient is the elastic relaxation process. Finally, we notice that for Si pillars as large as $50 \mu \mathrm{m}$ in width, the elastic relaxation effect is scarcely relevant for any Ge GR. In this case dislocations are found with a comparable distribution probability along the [001] growth direction as expected for a planar compositional graded layer [42].

\section{Conclusions}

We investigated the density, nature and distribution along the growth direction of dislocations in compositionally graded $\mathrm{SiGe}$ crystals epitaxially grown on micro-patterned $\mathrm{Si}(001)$ substrates by means of etch pits counting and TEM analysis. We demonstrate that by a proper choice of Si pillar width and Ge grading rate, coherent heterostructures were achieved. In this respect, compositionally graded 3D SiGe crystals appear much more attractive compared to analogous layered heterostructures where only the threading dislocation density can be controlled. Calculations of the nucleation energy of misfit dislocations agree with the experimental results. For dislocated crystals we analysed the fraction of misfit dislocations for different Si pillar width and Ge grading rate. We show that for shallow grading rate $\left(1.5 \% \mathrm{\mu m}^{-1}\right)$ and small Si pillar width, only misfit dislocations are found, while for higher grading rates their fraction is reduced due to the dislocation interactions. We provide also information about the distribution of dislocations along the [001] growth direction, demonstrating that by reducing the Si pillar width and Ge grading rate, dislocations are mostly located close to the $\mathrm{SiGe} / \mathrm{Si}$ heterointerface while the rest of the material is elastically relaxed.

\section{Acknowledgements}


We acknowledge the Sinergia project NOVIPIX CRSII2_147639 of the Swiss National Science

Foundation and Pilegrowth Tech srl for financial and technical support. We thank Dr. A. Marzegalli,

Dr. M. Meduňa, Prof. L. Miglio and Prof. B. Batlogg for fruitful discussions. We are grateful to Prof.

Gian-Luca Bona for continuous support and to the technical staff at the L-NESS laboratory in Como and FIRST clean room at the ETH in Zurich.

\section{References}

[1] Y. Kang, H.-D. Liu, M. Morse, M.J. Paniccia, M. Zadka, S. Litski, et al., Monolithic germanium/silicon avalanche photodiodes with $340 \mathrm{GHz}$ gain-bandwidth product, Nat. Photonics. 3 (2009) 59-63. doi:10.1038/nphoton.2008.247.

[2] Y. Tokuda, E. Yagyu, Silicon photonics: Look out III-V, Nat. Photonics. 3 (2009) 7-8. doi:10.1038/nphoton.2008.257.

[3] R. Soref, Mid-infrared photonics in silicon and germanium, Nat. Photonics. 4 (2010) 495-497. doi:10.1038/nphoton.2010.171.

[4] R. People, J.C. Bean, Calculation of critical layer thickness versus lattice mismatch for GeSi/Si strained-layer heterostructures, Appl. Phys. Lett. 47 (1985) 322-324.

[5] J.E. Ayers, New model for the thickness and mismatch dependencies of threading dislocation densities in mismatched heteroepitaxial layers, J. Appl. Phys. 78 (1995) 3724. doi:10.1063/1.359952.

[6] P.J. Taylor, W.A. Jesser, J.D. Benson, M. Martinka, J.H. Dinan, J. Bradshaw, et al., Optoelectronic device performance on reduced threading dislocation density GaAs/Si, J. Appl. Phys. 89 (2001) 4365-4375. doi:10.1063/1.1347000.

[7] M. Yamaguchi, A. Yamamoto, M. Tachikawa, Y. Itoh, M. Sugo, Defect reduction effects in GaAs on Si substrates by thermal annealing, Appl. Phys. Lett. 53 (1988) 2293. doi:10.1063/1.100257.

[8] Y. Yamamoto, G. Kozlowski, P. Zaumseil, B. Tillack, Low threading dislocation Ge on Si by combining deposition and etching, Thin Solid Films. 520 (2012) 3216-3221. doi:10.1016/j.tsf.2011.10.095.

[9] J.-S. Park, J. Bai, M. Curtin, B. Adekore, M. Carroll, A. Lochtefeld, Defect reduction of selective Ge epitaxy in trenches on $\mathrm{Si}(001)$ substrates using aspect ratio trapping, Appl. Phys. Lett. 90 (2007) 052113. doi:10.1063/1.2435603.

[10] J.Z. Li, J. Bai, J.-S. Park, B. Adekore, K. Fox, M. Carroll, et al., Defect reduction of GaAs epitaxy on $\mathrm{Si}(001)$ using selective aspect ratio trapping, Appl. Phys. Lett. 91 (2007) 21114. http://link.aip.org/link/?apl/91/021114.

[11] J. Bai, J.-S. Park, Z. Cheng, M. Curtin, B. Adekore, M. Carroll, et al., Study of the defect 
elimination mechanisms in aspect ratio trapping Ge growth, Appl. Phys. Lett. 90 (2007) 101902. doi:10.1063/1.2711276.

[12] K. Linthicum, T. Gehrke, D. Thomson, E. Carlson, P. Rajagopal, T. Smith, et al., Pendeoepitaxy of gallium nitride thin films, Appl. Phys. Lett. 75 (1999) 196. doi:10.1063/1.124317.

[13] C. V. Falub, H. von Känel, F. Isa, R. Bergamaschini, A. Marzegalli, D. Chrastina, et al., Scaling Hetero-Epitaxy from Layers to Three-Dimensional Crystals, Science 335 (2012) 1330-1334. doi:10.1126/science.1217666.

[14] A. Marzegalli, F. Isa, H. Groiss, E. Müller, C. V Falub, A.G. Taboada, et al., Unexpected Dominance of Vertical Dislocations in High-Misfit Ge/Si(001) Films and Their Elimination by Deep Substrate Patterning, Adv. Mater. 25 (2013) 4408-4412. doi:10.1002/adma.201300550.

[15] F. Isa, A. Marzegalli, A.G. Taboada, C. V. Falub, G. Isella, F. Montalenti, et al., Onset of vertical threading dislocations in $\mathrm{Si}_{1-x} \mathrm{Ge}_{x} \mathrm{Si}(001)$ at a critical Ge concentration, APL Mater. 1 (2013) 052109. doi:10.1063/1.4829976.

[16] E.A. Fitzgerald, Y.-H. Xie, M.L. Green, D. Brasen, A.R. Kortan, J. Michel, et al., Totally relaxed $\mathrm{Ge}_{x} \mathrm{Si}_{1-x}$ layers with low threading dislocation densities grown on Si substrates, Appl. Phys. Lett. 59 (1991) 811-813. doi:10.1063/1.105351.

[17] A.Y. Kim, W.S. McCullough, E.A. Fitzgerald, Evolution of microstructure and dislocation dynamics in $\operatorname{In}_{x} \mathrm{Ga}_{1-x} \mathrm{P}$ graded buffers grown on $\mathrm{GaP}$ by metalorganic vapor phase epitaxy: Engineering device-quality substrate materials, J. Vac. Sci. Technol. B. 17 (1999) 1485-1501. http://link.aip.org/link/?jvb/17/1485.

[18] Y.B. Bolkhovityanov, O.P. Pchelyakov, M.A. Revenko, L. V Sokolov, S.I. Chikichev, Artificial GeSi Substrates for Heteroepitaxy: Achievements and Problems, Semiconductors. 37 (2003) 493-518. http://dx.doi.org/10.1134/1.1575352.

[19] S. Luryi, E. Suhir, New approach to the high quality epitaxial growth of lattice-mismatched materials, Appl. Phys. Lett. 49 (1986) 140-142. doi:10.1063/1.97204.

[20] A. Atkinson, S.C. Jain, A.H. Harker, Strain, dislocations, and critical dimensions of laterally small lattice- mismatched semiconductor layers, J. Appl. Phys. 77 (1995) 1907-1913. http://link.aip.org/link/?jap/77/1907.

[21] D. Zubia, S.H. Zaidi, S.D. Hersee, S.R.J. Brueck, Nanoheteroepitaxy: Nanofabrication route to improved epitaxial growth, J. Vac. Sci. Technol. B. 18 (2000) 3514. doi:10.1116/1.1321283.

[22] F. Montalenti, M. Salvalaglio, A. Marzegalli, P. Zaumseil, G. Capellini, T.U. Schülli, et al., Fully coherent growth of Ge on free-standing $\mathrm{Si}(001)$ nanomesas, Phys. Rev. B. 89 (2014) 014101. doi:10.1103/PhysRevB.89.014101.

[23] G. Niu, C. Giovanni, G. Lupina, T. Niermann, M. Salvalaglio, A. Marzegalli, et al., Photodetection in hybrid single layer graphene/fully coherent $\mathrm{Ge}$ island nanostructures selectively grown on Si nano-tip patterns, ACS Appl. Mater. Interfaces. 8 (2016) 2017-2026. doi:10.1021/acsami.5b10336.

[24] X.W. Zhou, D.K. Ward, F.P. Doty, J.A. Zimmerman, B.M. Wong, J.L. Cruz-Campa, et al., A prediction of dislocation-free $\mathrm{CdTe} / \mathrm{CdS}$ photovoltaic multilayers via nano-patterning and composition grading, Prog. Photovoltaics Res. Appl. 23 (2015) 1837-1846. doi:10.1002/pip.2628.

[25] F. Isa, M. Salvalaglio, Y.A.R. Dasilva, M. Meduňa, M. Barget, A. Jung, et al., Highly 
Mismatched, Dislocation-Free SiGe/Si Heterostructures, Adv. Mater. 28 (2016) 884-888. doi:10.1002/adma.201504029.

[26] C. Rosenblad, H.R. Deller, A. Dommann, T. Meyer, P. Schroeter, H. von Känel, Silicon epitaxy by low-energy plasma enhanced chemical vapor deposition, J. Vac. Sci. Technol. A. 16 (1998) 2785-2790. http://link.aip.org/link/?jva/16/2785.

[27] M. Salvalaglio, F. Montalenti, Fine control of plastic and elastic relaxation in Ge/Si vertical heterostructures, J. Appl. Phys. 116 (2014) 104306. doi:10.1063/1.4895486.

[28] R. Bergamaschini, F. Isa, C.V. Falub, P. Niedermann, E. Müller, G. Isella, et al., Self-aligned Ge and SiGe three-dimensional epitaxy on dense Si pillar arrays, Surf. Sci. Rep. 68 (2013) 390-417. doi:10.1016/j.surfrep.2013.10.002.

[29] L.D. Landau, E.M. Lifshitz, Theory of Elasticity, 1970. doi:10.1063/1.3057037.

[30] R. Gatti, A. Marzegalli, V. Zinovyev, F. Montalenti, L. Miglio, Modeling the plastic relaxation onset in realistic SiGe islands on Si(001), Phys. Rev. B. 78 (2008) 184104. http://link.aps.org/abstract/PRB/v78/e184104.

[31] P. Hirth, J. Lothe, Theory of Dislocations, McGraw-Hill, New York, 1985.

[32] W. Cai, A. Arsenlis, C.R. Weinberger, V. V. Bulatov, A non-singular continuum theory of dislocations, J. Mech. Phys. Solids. 54 (2006) 561-587. doi:10.1016/j.jmps.2005.09.005.

[33] R. Gatti, F. Boioli, M. Grydlik, M. Brehm, H. Groiss, M. Glaser, et al., Dislocation engineering in SiGe heteroepitaxial films on patterned Si (001) substrates, Appl. Phys. Lett. 98 (2011) 121908. doi:10.1063/1.3569145.

[34] P.M.J. Maree, J.C. Barbour, J.F. van deer Veen, K.L. Kavanagh, C.W.T. Bulle-Lieuwma, M.P.A. Viegers, Generation of misfit dislocations in semiconductors, J. Appl. Phys. 62 (1987) 4413.

[35] C.W. Leitz, M.T. Currie, A.Y. Kim, J. Lai, E. Robbins, E.A. Fitzgerald, et al., Dislocation glide and blocking kinetics in compositionally graded SiGe/Si, J. Appl. Phys. 90 (2001) 2730. doi:10.1063/1.1389333.

[36] J.S. Speck, M.A. Brewer, G. Beltz, A.E. Romanov, W. Pompe, Scaling laws for the reduction of threading dislocation densities in homogeneous buffer layers, J. Appl. Phys. 80 (1996) 38083816. http://link.aip.org/link/?jap/80/3808.

[37] S. Castellanos, M. Kivambe, J. Hofstetter, M. Rinio, B. Lai, T. Buonassisi, Variation of dislocation etch-pit geometry: An indicator of bulk microstructure and recombination activity in multicrystalline silicon, J. Appl. Phys. 115 (2014) 183511. doi:10.1063/1.4876445.

[38] W.N. Borle, R.K. Bagai, Dislocation etch pits on various crystal planes of silicon, J. Cryst. Growth. 36 (1976) 259-262. doi:10.1016/0022-0248(76)90286-4.

[39] K.H. Yang, An Etch for Delineation of Defects in Silicon, J. Electrochem. Soc. 131 (1984) 1140. doi:10.1149/1.2115767.

[40] K. Schwarz, Interaction of Dislocations on Crossed Glide Planes in a Strained Epitaxial Layer, Phys. Rev. Lett. 78 (1997) 4785-4788. doi:10.1103/PhysRevLett.78.4785.

[41] A. Sakai, T. Tatsumi, K. Aoyama, Growth of strain-relaxed Ge films on Si(001) surfaces, Appl. Phys. Lett. 71 (1997) 3510. doi:10.1063/1.120375.

[42] J. Tersoff, Dislocation and strain relief in compositionally graded layers, Appl. Phys. Lett. 62 
(1993) 693-695. http://link.aip.org/link/?apl/62/693.

[43] K.W. Schwarz, Simulation of dislocations on the mesoscopic scale. II. Application to strainedlayer relaxation, J. Appl. Phys. 85 (1999) 120-129. doi:10.1063/1.369430.

[44] F.K. LeGoues, B.S. Meyerson, J.F. Morar, P.D. Kirchner, Mechanism and conditions for anomalous strain relaxation in graded thin films and superlattices, J. Appl. Phys. 71 (1992) 4230-4243. http://link.aip.org/link/?jap/71/4230.

\section{List of figure captions:}

Figure 1: Scheme of the 2D crystal geometry indicating the parameters and BCs used for the FEM calculations. The indexes $L, N$ and $i$ represent the crystal width, the $\mathrm{SiGe}$ layer number and the interface number, respectively. The Si substrate is five times taller and wider than the Si pillar.

Figure 2: a) Top view SEM image of SiGe crystals $\left(\mathrm{GR}=3 \% \mu \mathrm{m}^{-1}\right)$ on $5 \times 5 \mu \mathrm{m}^{2}$ Si pillars. b) Crosssectional SEM image of a SiGe crystal $\left(\mathrm{GR}=1.5 \% \mu \mathrm{m}^{-1}\right)$ on a Si pillar $2 \mu \mathrm{m}$ in width. SEM images of the $\mathrm{Si}_{1-x} \mathrm{Ge}_{x}$ with $x=0.5 \%$ constant composition alloy and of the bare $\mathrm{Si}$ pillar are also superimposed. $L_{1}$ and $L_{2}$ indicate the width of the $x=0.5 \%$ constant composition alloy and of the $\mathrm{Si}_{0.6} \mathrm{Ge}_{0.4}$ capping layer, respectively. c) Cross-sectional SEM image of a SiGe crystal $\left(\mathrm{GR}=1.5 \% \mu \mathrm{m}^{-1}\right)$ after defect etching in the $\mathrm{Cr}$ based solution for $240 \mathrm{~s}$. Etch pits related to dislocations are visible in the $\mathrm{SiGe}$ crystal (orange arrow) and in the Si pillar (purple arrow). The red dashed line indicates the heterointerface which is well defined by the vanishing of the Bosch ripples related to the Si pillar fabrication process [28].

Figure 3: a) Probability distribution of the dislocation density in the $8 \mu \mathrm{m}$ thick $\mathrm{Si}_{1-x} \mathrm{Ge}_{x} x=0.5 \%$ constant composition crystals for different Si pillar width (see coloured spheres). The dislocation density was evaluated considering the region over the red dashed line of figure $2 \mathrm{c}$ ). The dislocation density in the unpatterned film-like area is indicated in dark blue $\left.\left.\left(\mathrm{DD}_{\text {unpat }}\right) . \mathrm{b}\right), \mathrm{c}\right)$ and $\left.\mathrm{d}\right)$ are the same of 
a) but for SiGe crystals grown with a Ge GR $=1.5,3$ and $6 \% \mu \mathrm{m}^{-1}$, respectively. In b), c) and d) the dislocation density was measured including both the constant Ge composition $(x=0.5 \%)$ and graded part. In b) the black arrow highlights the data point referring to dislocation-free SiGe crystals deposited with a GR $=1.5 \% \mu \mathrm{m}^{-1}$ on $2 \times 2 \mu \mathrm{m}^{2}$ Si pillars. The error bar on the abscissa is $\pm 20 \%$.

Figure 4: Formation energy $\Delta E_{\min }$ of a $60^{\circ} \mathrm{MD}$ at the $i=1$ interface calculated as a function of the number of $\mathrm{Si}_{1-x} \mathrm{Ge}_{x}$ layers $N$ for three different $\mathrm{SiGe} / \mathrm{Si}$ crystal systems: $L=5.9 \mu \mathrm{m}$ and $\mathrm{GR}=1.5 \% \mu \mathrm{m}^{-1}$ (solid black line), $L=7.0 \mu \mathrm{m}$ and $\mathrm{GR}=1.5 \% \mu \mathrm{m}^{-1}($ dashed black line $), L=5.9 \mu \mathrm{m}$ and $\mathrm{GR}=3 \% \mu \mathrm{m}^{-1}$ (dotted black line).

Figure 5: a) Cross-sectional SEM image of a SiGe crystal with Ge GR $=3 \% \mu \mathrm{m}^{-1}$ deposited on a $5 \times 5$ $\mu \mathrm{m}^{2}$ Si pillar after defect etching. Examples of TD and MD are indicated by a red and light blue arrow, respectively. b) and c) are magnified SEM images of etch pits related to TDs and MDs, respectively. d) Fraction of MDs in SiGe crystals grown with a Ge GR of $1.5 \% \mu \mathrm{m}^{-1}$ (purple), $3 \% \mu \mathrm{m}^{-1}$ (orange) and $6 \% \mu^{-1}$ (grey) as a function of the Si pillar width. The MD fraction is defined as the number of MDs over the number of TDs + MDs. The purple star indicates the fraction of MDs obtained from a TEM analysis close to the Si heterointerface of three SiGe crystals deposited at the GR of $1.5 \% \mu^{-1}$ on $5 \times 5$ $\mu \mathrm{m}^{2}$ Si pillars.

Figure 6: a) Cross-sectional SEM image after defect etching of SiGe crystals with Ge GR $=3 \% \mu \mathrm{m}^{-1}$ on $\mathrm{Si}$ pillars $5 \mu \mathrm{m}$ in width. The $\mathrm{SiGe} / \mathrm{Si}$ structure is divided in 6 regions to evaluate the distribution of dislocations along the [001] direction. The first region consists of the Si pillars (blue dashed line), the second one of the $8 \mu \mathrm{m}$ thick SiGe alloy with constant Ge content $x=0.5 \%$ (purple dashed lines). The other 4 regions are defined by a Ge content varying by $\delta x=0.1$ from region to region (orange dashed 
lines). b) Distribution of dislocations along the [001] direction in the compositionally graded part of SiGe crystals with Ge GR $=1.5 \% \mu \mathrm{m}^{-1}$ on Si pillars with different width. c) and d) are the same as b) but for SiGe crystals grown with a Ge GR of 3 and $6 \% \mu^{-1}$, respectively. In b), c) and d) the value of the Ge content $x$ shown on the bottom abscissa refers to the highest Ge content in each region.

Analogously the reported $Z$ values on the upper abscissa indicate the height at which $\mathrm{Si}_{1-x} \mathrm{Ge}_{x}$ alloys with $x=0.5 \%, 0.1,0.2,0.3$ and 0.4 are located. 
Figure 1

$\widehat{Z}[001]$

Si Pillar

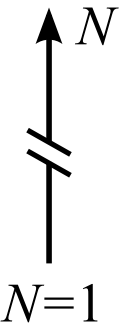

SiGe
$=0.5 \%$

$i=0$



free BC

Si Substrate $\quad \mathrm{u}_{\mathrm{x}}=0$

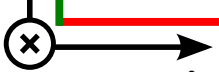

$\hat{\boldsymbol{Y}}[\overline{110]} \quad \hat{\boldsymbol{X}}[110]$ 







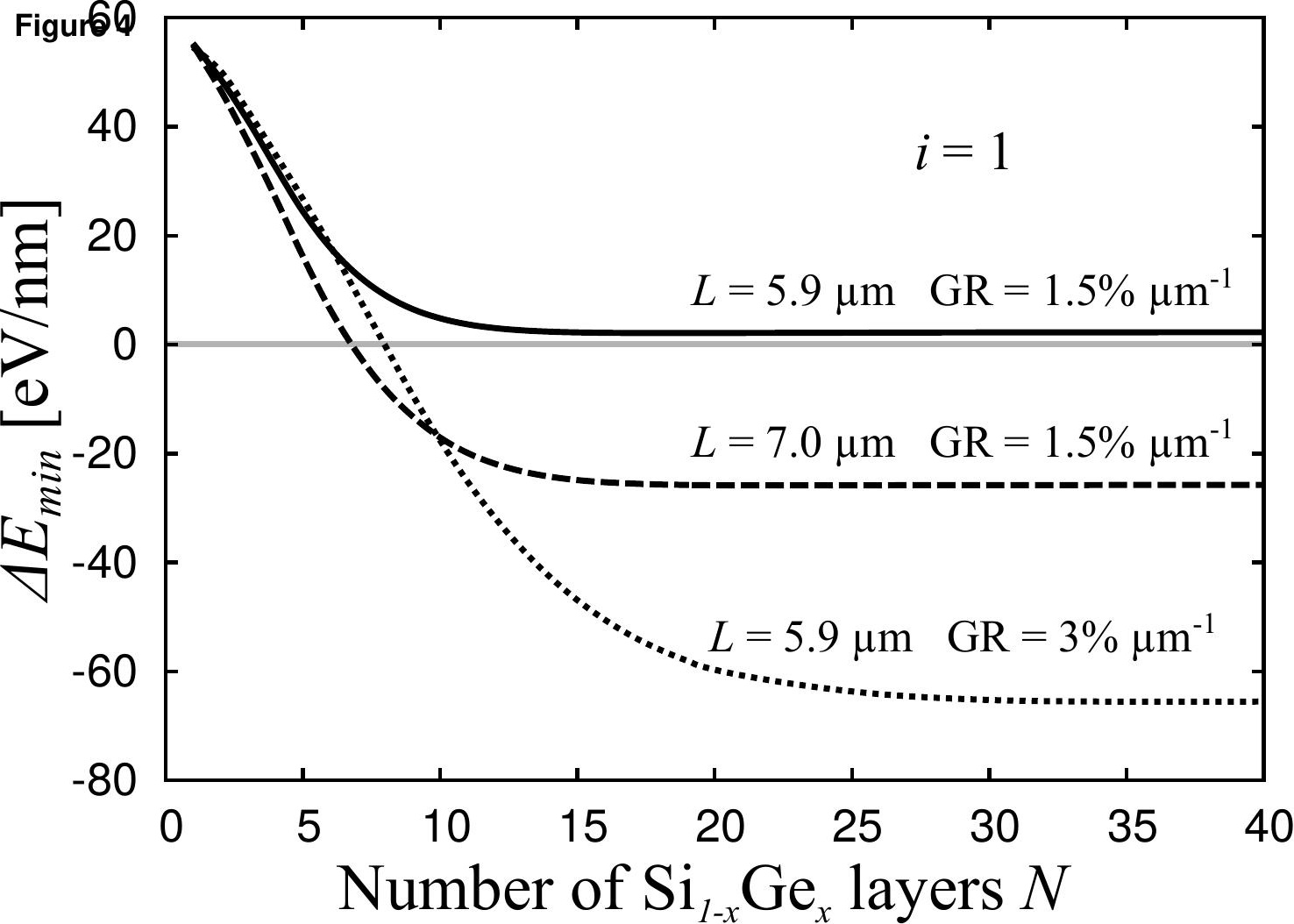




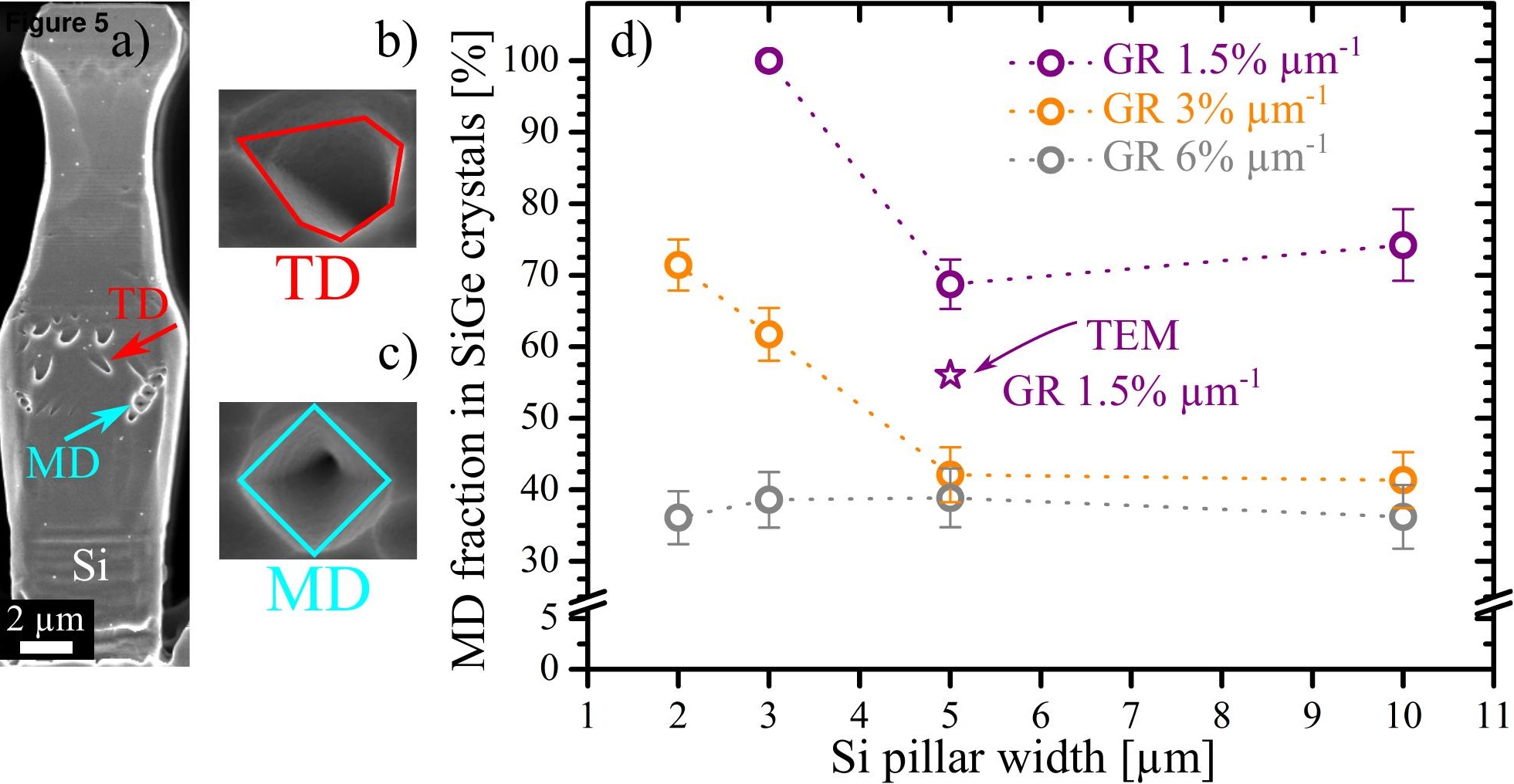




\section{Figure 6}

a)

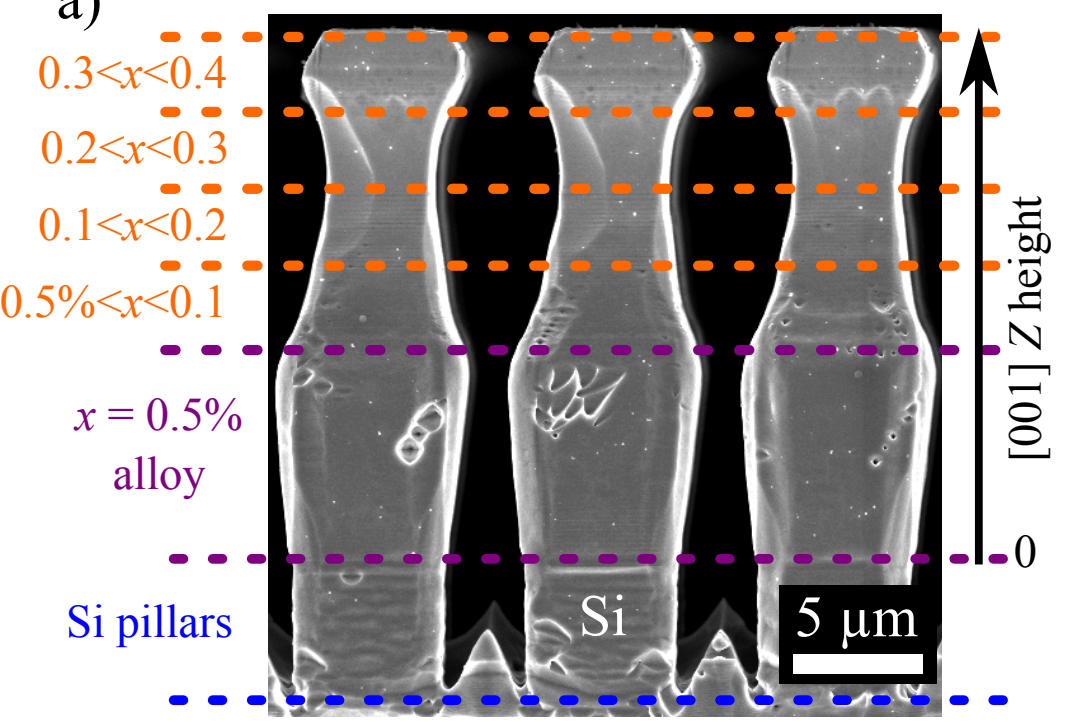

[001] $Z$ height $[\mu \mathrm{m}]$
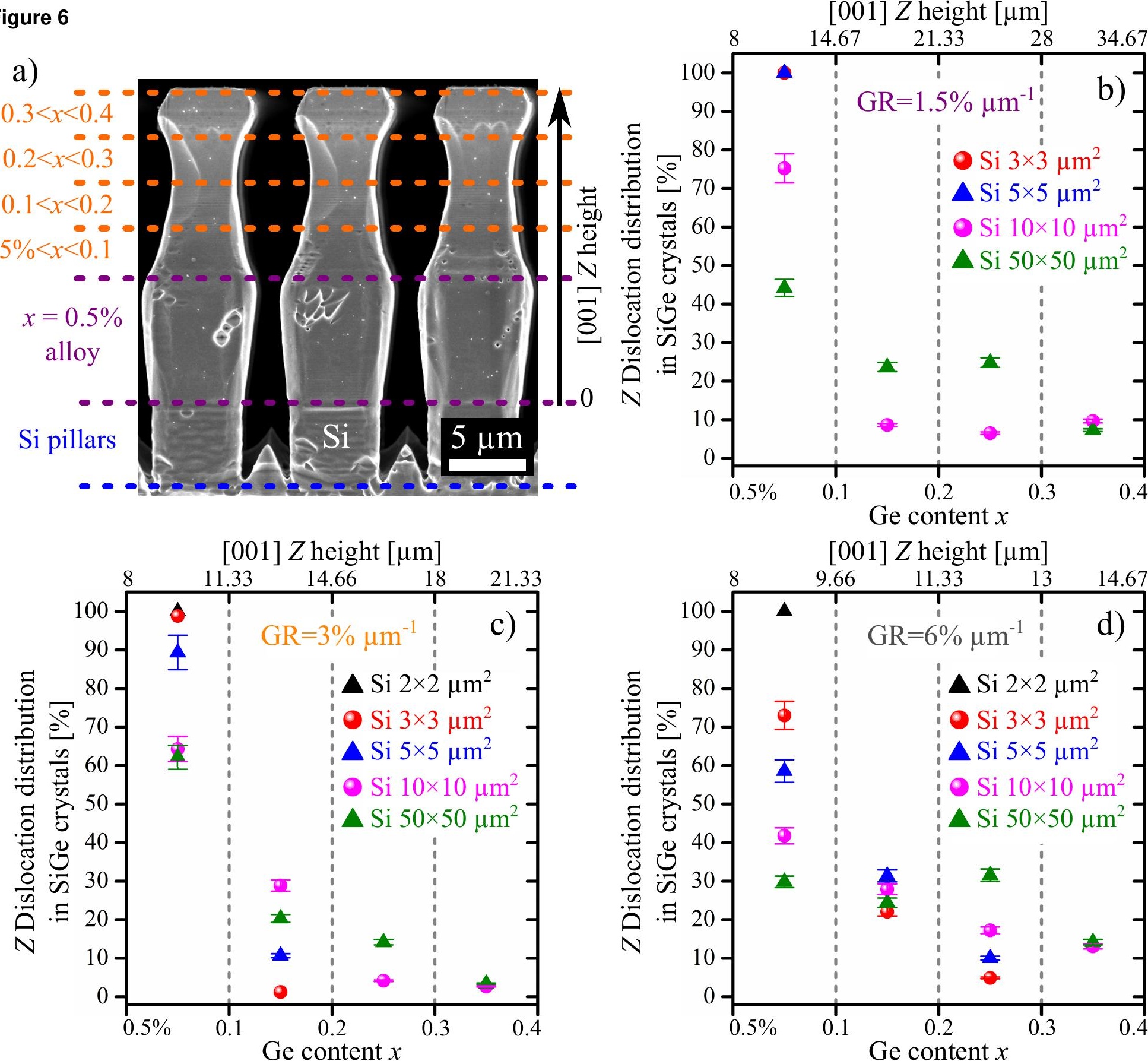


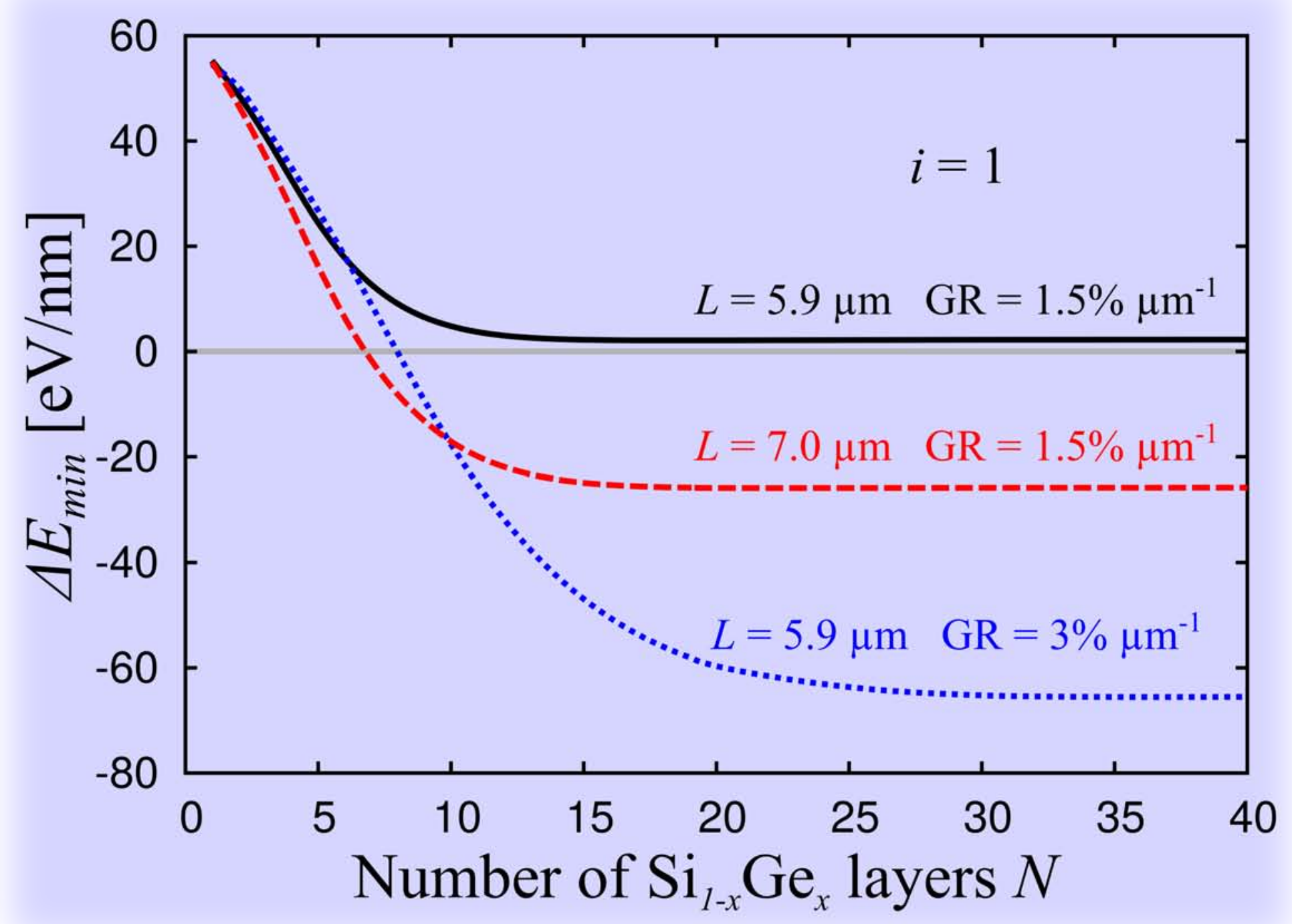

